\title{
MULTI-RESONANCE AND ENHANCED SYNCHRONIZATION IN STOCHASTICALLY COUPLED RATCHETS
}

\author{
B. R. NANA NBENDJO \\ Laboratory of Modelling and Simulation in Engineering and Biological Physics, \\ Faculty of Sciences, University of Yaoundé I, PO Box 812 Yaoundé, Cameroon \\ nananbendjo@yahoo.com
}

Max-Planck-Institut für Physik komplexer Systeme Nüthnitzer Str. 38, D-01187 Dresden, Germany U. E. VINCENT

Department of Physics, Lancaster University, Lancaster, LA1 4YB, UK

u.vincent@lancaster.ac.uk

Department of Physics, Olabisi Onabanjo University, Ago-Iwoye, Nigeria

PETER V.E. McCLINTOCK

Department of Physics, Lancaster University, Lancaster, LA1 $4 Y B, U K$

p.v.e.mcclintock@lancaster.ac.uk

Received (to be inserted by publisher)

\begin{abstract}
We investigate the dynamics and synchronization of two inertia ratchets interacting indirectly through a stochastic dynamical environment. We examine resonant oscillations in their synchronous and asynchronous modes; and we determine the effects of the interaction with the environment on the system's response and synchronization. We show that noise-induced multiresonance and noise-enhanced synchronization emerge from the ratchets' interaction with their noisy environment. The simultaneous quenching of the chaotic regimes, and the domain of gain parameters for efficient control, are identified. It is shown that optimal transport can be achieved, implying that an inertia ratchet can take advantage of its noisy environment to enhance its rich dynamical and transport properties.
\end{abstract}

Keywords: Stochastic ratchets; Synchronization; Chaos; Multiresonance

\section{Introduction}

The non-equilibrium dynamics of particles in a ratchet potential (i.e. a periodic potential that lacks reflection symmetry) has long been considered a problem of fundamental importance in statistical physics [Hänggi \& Bartussek, 1996; Astumian, 1997; Jülicher et al., 1997; Reimann et al., 1997]. It has become the subject of strong research focus recently on account of its relevance to diverse areas of physics, chemistry and biology. An important characteristic of a ratchet system is that, when it is subjected to a stationary non-equilibrium perturbation, particle motion in one particular direction is favoured. Several different models have been introduced in attempts to understand the generation of unidirectional motion from such non-equilibrium fluctuations. They have been classified by Hänggi \& Bartussek [1996]. Among them, our 
interest centres on the rocking ratchet system, in which the particles move in an asymmetric periodic potential subject to spatially-uniform, time-periodic forces of zero average. In one example, Jung et al. [1996] studied the effect of finite inertia in a deterministically-rocked periodic potential. They considered the non-stochastic deterministic case in which noise is absent, thus allowing for the possibility of obtaining chaotic as well as regular dynamics. In some respects, this deterministically induced chaos can mimic the role of noise, thereby steering the system to exhibit current flow in either direction. Following this work, Mateos [2000] established a relationship between the bifurcation and transport properties. In particular, he showed that current reversals occur at critical bifurcation points associated with type-I intermittency [Son et al., 2003], arousing wide interest [Barbi \& Salerno, 2000; Arizmendi et al., 2001; Al-Khawaja, 2005; Li, 2006; Kenfack et al., 2007]

Quite generally, a coupling between two systems can result in synchronization, and it has been shown that a coupling between ratchets can cause them to synchronize [Vincent et al., 2004, 2005; Kostur et al., 2005; Vincent \& Laoye, 2007b,a; Lu et al., 2008; Mateos \& Alatriste, 2008; Zarlenga et al., 2009; Xu et al., 2009; Vincent et al., 2010b,a]. The resulting synchrony has implications for their transport properties and particularly for the control of directed transport [Vincent \& Laoye, 2007b; Xu et al., 2009; Lu et al., 2008]. Previous synchronization studies assumed that the two ratchets interact directly via non-stochastic linear or nonlinear couplings. In the context of interactions in some physical and living systems, there is evidence, however, that the coupling may not always be direct [Steele et al., 2003; Strogatz et al., 2005; Taylor et al., 2009; Zamora-Munt et al., 2010; Fischer et al., 2006]. For instance, populations of cells, e.g. suspensions of yeast in nutrient solutions, may undergo transitions to coordinated activity that is believed to arise through communication via chemicals diffusing in the surrounding medium [Katriel, 2008; Taylor et al., 2009]. Similarly, walkers coupled to the oscillations of the Millennium bridge achieved synchrony with one another when the number of walkers on the bridge was sufficiently large [Strogatz et al., 2005; ZamoraMunt et al., 2010]. Another situation is found in semiconductors, where some authors have considered a system of semiconductor lasers coupled to a central laser with and without time-delays [Fischer et al., 2006; Zamora-Munt et al., 2010]. These studies suggest that an indirect channel could be used to couple systems, such that the dynamics of all the systems might simultaneously be modified by tuning the physical characteristics of the channel. Such an interaction scheme has also been used to demonstrate the role played by indirectly coupled oscillators in circadian rhythms [Camacho et al., 2004; Rompala et al., 2007].

Moreover, it is well known that noise is ubiquitous in both artificial and natural systems [Anishchenko et al., 2007; Schimansky-Geier et al., 2003; Zhou et al., 2002; Zhou \& Kurths, 2002]. Thus, the interactions between synchronizing systems are also unavoidably subject to the effects of stochastic perturbations. In this direction, noise-induced and noise-enhanced resonances and synchronization have been reported in linearly coupled chaotic systems [Maritan \& Banavar, 1994; Zhou et al., 2002; Zhou \& Kurths, 2002; García-Álvarez et al., 2009; Kenfack \& Singh, 2010; Senthilkumar \& Kurths, 2010]. Zhou et al. [2002] showed both numerically and experimentally that noise can enhance phase synchronization; and in [Zhou \& Kurths, 2002], noise-induced phase synchronization and its mechanism for nonidentical chaotic systems driven by common noise was revealed. Recently, García-Álvarez et al. [2009] showed that noise and (direct) coupling are often competing factors in the induction of synchronization; and their coexistence can inhibit the synchronization independently induced by either. The synchronization of noisy-coupled chaotic systems has also been investigated from nonlinear control approaches [Lin \& He, 2005; Chen, 2007; Tang et al., 2008; Sun \& Cao, 2007a,b].

In the light of the foregoing, we investigate in this paper the effects of noisy coupling on the dynamics and synchronization of two inertia ratchets that are coupled via a stochastic dynamical environment. We will show that resonance oscillations that exist in the absence of coupling are destroyed when the ratchets interact via a noiseless channel. However, the gradual activation of noise induces multiple resonances. It is also shown that synchronization can be induced and enhanced in the weakly-coupled regime where synchrony does not occur in the noiseless case. Furthermore, we explore the dynamical environment to control the underlying chaotic dynamics and transport property by choosing appropriate coupling gain; and we show that optimal transport may be achieved even in the presence of noise. The results that we present below show that a noisy environment can significantly enhance the performance of a system. The rest of the paper is organized as follows. In Sec. 2, we describe the model. In Sec. 3, we examine 
the resonance oscillations and synchronization for both the noiseless and noisy cases. The corresponding influence of the environment on chaos and on the transport properties are discussed in Sec. 4. The paper is concluded in Sec. 5 .

\section{Ratchet Model}

We consider a deterministic particle moving in an asymmetric potential while being driven by a zeroaverage, time-dependent, periodic external force. The dynamics is exclusively deterministic. Thus we consider a deterministically rocked ratchet that satisfies the following dimensionless inertial dynamics [Mateos, 2000],

$$
\ddot{s}+\lambda \dot{s}+\frac{d v(s)}{d s}=F_{0} \cos (\omega t)
$$

where $v(s)$ is the asymmetric ratchet potential, and $F_{0}$ and $\lambda$ are the forcing strength and the damping parameter, respectively. The period associated with the frequency $\omega$ of the linear motion around the minima of the ratchet potential is used as the natural unit in which to scale time, implying that all quantities are in dimensionless units. Thus the dimensionless potential $v(s)$ is given by

$$
v(s)=C-\frac{1}{4 \pi^{2} \delta}\left[\sin 2 \pi\left(s-x_{0}\right)+0.25 \sin 4 \pi\left(s-x_{0}\right)\right]
$$

where $C \simeq 0.0173$ and $\delta \simeq 1.6$ are fixed potential parameters. The periodic potential $v(s)$ has an infinite number of potential wells as shown in Fig. 1 . It is shifted by a value $x_{0}$ in order to place one of the minima at the origin.

The dynamics and transport of the single ratchet system (1) and (2) have been investigated when under the influence of noise [Arizmendi et al., 2001; Li, 2006]. Arizmendi et al. [2001] showed that the consequence of disorder (noise) is the appearance of current reversal and chaotic diffusion on regular trajectories; whereas on some chaotic trajectories disorder induces regular motion. Similarly, Li [2006] showed that at low noise the transport is mainly chaotic and at high temperature it is mainly stochastic. Here, we consider two inertia ratchets (1) coupled via a noisy channel. The channel is a stochastic and dynamical environment which provides feedback into the systems, through a gain. The system is simply expressed as

$$
\begin{aligned}
& \ddot{x}+\lambda \dot{x}+\frac{d v(x)}{d x}=F_{0} \cos \omega t+k(z-x) \\
& \ddot{y}+\lambda \dot{y}+\frac{d v(y)}{d y}=F_{0} \cos \omega t+k(z-y) \\
& \dot{z}=k(x-z)+k(y-z)+\xi(t)
\end{aligned}
$$

with

$$
v(\cdot)=C-\frac{1}{4 \pi^{2} \delta}\left[\sin 2 \pi\left((\cdot)-x_{0}\right)+0.25 \sin 4 \pi\left((\cdot)-x_{0}\right)\right],
$$

where the stochastic term $\xi(t)$ is Gaussian white noise of zero mean (i.e. $\langle\xi(t)\rangle=0$ and $\left.\left\langle\xi(t) \xi\left(t^{\prime}\right)\right\rangle=0\right)$ and correlation $\left\langle\xi(t) \xi\left(t^{\prime}\right)\right\rangle=2 D \delta\left(t-t^{\prime}\right)$, with $D$ being the intensity of the noise. $v(\cdot)$ is the asymmetric periodic potential, $k$ the coupling coefficient or control gain parameter and other quantities are as in Eq. (1). Notice that the characteristics of our system (3) are such that: (i) the two ratchets are not directly coupled to one another, rather, the interaction is provided by a common stochastic dynamical environment; (ii) the environment (with state space $z$ ) is dynamical and stochastic while each ratchet $(x, y)$ is deterministic; and (iii) when $D=0$ the dynamics of system (3) is deterministic. Since the coupling environment consists of both deterministic and stochastic dynamics, the parameters $k$ and $D$ play key roles in the systems' dynamics. In the limit $D \rightarrow 0$, deterministic dynamics predominates while in the limit $D \rightarrow \infty$, stochastic effects predominate. Although $D$ may assume the value zero, $k$ remains nonzero so long as the oscillators interact with the environment.

We point out that our system (3) could be useful in the description of certain biological systems, such as two cells in which oscillatory reactions take place through communication via chemicals diffusing 


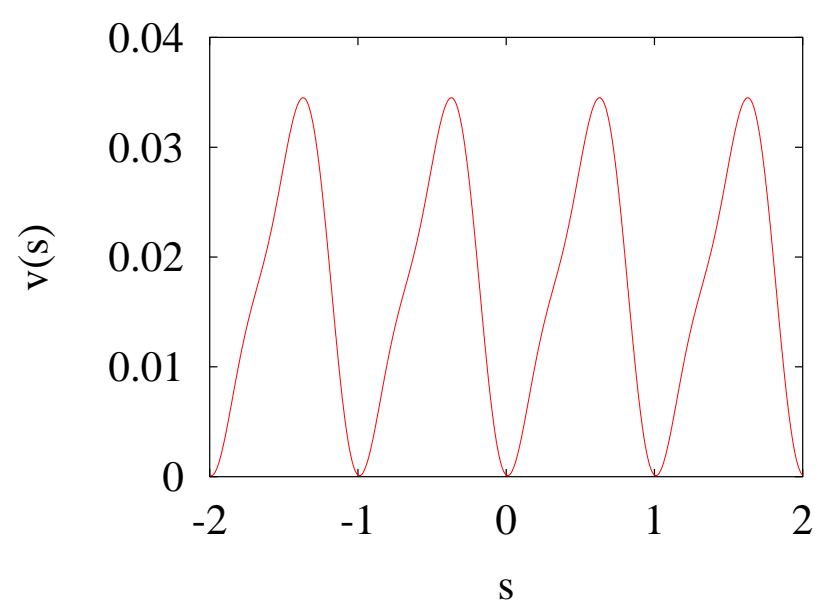

Fig. 1. The ratchet potential (2) for $C=0.0173, \delta \simeq 1.6$ and $x_{0}=-0.19$

within the surrounding medium, wherein the generation of macroscopic oscillations is determined by the ability of the cells to synchronize with each other or, as we consider here, when each of the systems synchronizes separately with their common environment [Katriel, 2008]. Extending the purely deterministic configurations earlier presented by Katriel [2008], Strogatz et al. [2005], Zamora-Munt et al. [2010], Fischer et al. [2006] and Zamora-Munt et al. [2010], our model also assumes that the common environment may be under the influence of noise, because noise is of course omnipresent in real life situations.

\section{Synchronization and Resonance Oscillations}

\subsection{Synchronous mode}

Let us first consider the channel to be a noiseless environment, that is, $D=0$. In this case, the two systems could be in either a synchronous or an asynchronous mode. We define the full synchronization mode for $D=0$ as a periodic motion satisfying the condition: $x(t)=y(t)=s(t)$. Based on this assumption we can, by adding the first two expressions in (3), obtain the following system characterizing the synchronized state

$$
\begin{aligned}
& \ddot{s}+\lambda \dot{s}+\frac{d v(s)}{d s}=F_{0} \cos (\omega t)+k(z-s), \\
& \dot{z}=2 k(s-z),
\end{aligned}
$$

with $v(s)$ given by equation (2). The stability of the synchronized dynamics given by Eq. (5) can easily be verified by using Lyapunov stability theory and Linear Matrix Inequalities (LMI), as we have recently shown [Vincent et al., 2010b]. Resmi et al. [2010] employed a method based on Lyapunov exponents, and this can readily be applied by making appropriate approximations like those used by Vincent et al. [2010b]. We leave this stability analysis for verification by the reader. An important feature of the synchronized dynamics given by Eq. (5) is the non-vanishing property of the interaction term as found in elastically coupled oscillators [Vincent et al., 2010b,a] where the coupling vanishes in the synchronized state. Here, in contrast, the ratchets never decouple, implying that there is always a constant in-and-out flow of energy from the channel to-and-from each of the systems. We focus our attention on the effect of this control on the periodic oscillations and synchronization dynamics. We examine in Fig. 2 the dependence of the amplitude of vibration on the external forcing amplitude for both the coupled and uncoupled systems and for different driving frequencies $(\omega)$. We found that, for a given frequency, there is a forcing amplitude $F_{0}$ at which the amplitude of vibration attains its peak value. As $\omega$ increases, larger $F_{0}$ is required for the system to vibrate at maximum amplitude as depicted in Fig. 2(b). Notably, for $\omega=0.5$, the peak occurs at $F_{0} \approx 0.24$ as shown in $2(\mathrm{a})$ and for higher driving frequencies, the peaks occur at $F_{0} \approx 0.49,0.59,0.73$ and 0.83 for $\omega=0.6,0.7,0.8$ and 0.9 , respectively. Furthermore, Fig. 2(a) shows that the coupling strength 

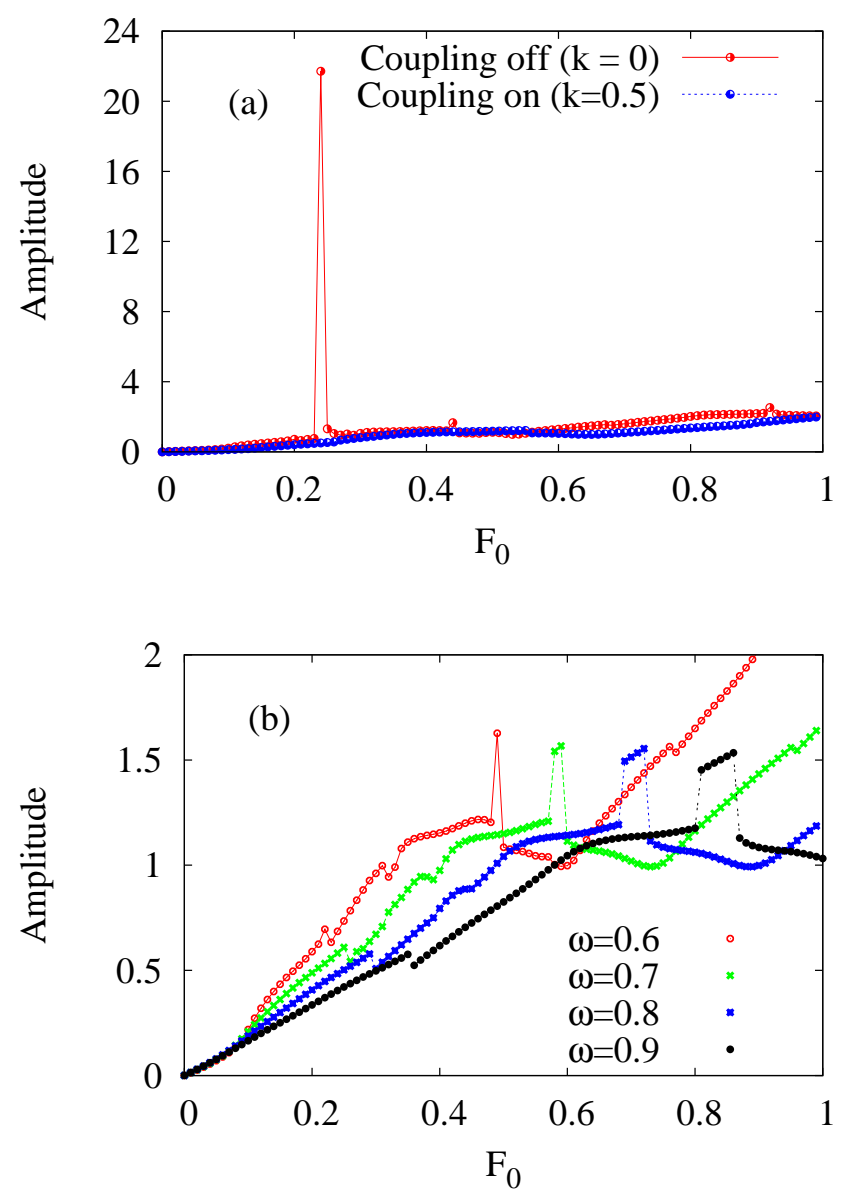

Fig. 2. Amplitude of harmonic oscillation in $x(t)$ as a function of $F_{0}$ showing (a) a resonant peak for $\lambda=0.75$, and $\omega=0.5$ at $F_{0}=0.24$ when uncoupled $(k=0 ; D=0)$ and its disappearance when coupled $(k=0.5 ; D=0)$; and (b) for higher driving frequencies $\omega=0.6,0.7,0.8$, and 0.9 when uncoupled.

has a profound effect on the system's response. For instance, for $k=0.5$ the resonant response that occurs in the uncoupled system disappears completely. This effect was also observed for other frequencies.

To examine the effect of the control gain parameter $k$ on the synchronization process, we first show in Fig. 3 the system's response to the control gain parameter for $F_{0}=0.5$; that for the uncontrolled case, $k=0$ is indicated by the horizontal line. For $k \geq 0.1$ the system undergoes a rapid decrease in its response as $k$ increases, reaching a minimum at $k=0.5$; and thereafter attaining steady-state. This implies that, by making a suitable choice of $k$ one can optimize the channel for efficient transport control. To ascertain whether or not this maximum and minimum correspond to synchronization transitions, we can make use of the error state $L$, an index that provides a good measure of the synchronization quality. The error state for a given trajectory over a long time dynamics $T$, is given by:

$$
L=\frac{1}{T} \int_{0}^{T}\left[\left(x_{2}-x_{1}\right)^{2}+\left(\dot{x}_{2}-\dot{x}_{1}\right)^{2}\right]^{1 / 2} d t
$$

In general, if $L^{2} \rightarrow 0$ or $L^{2} \leq \epsilon$, where $\epsilon$ is some small tolerance (say, 0.005), then the coupled ratchets may be assumed to have attained their fully synchronized state. In Fig. 4, we have plotted $L^{2}$ and $x(t)$ as functions of $k$. For $k \geq k_{c}=0.08, L^{2} \rightarrow 0$ indicating full synchrony. Near $k_{c}$, the system oscillation is maximum, implying that the oscillation resonance in Fig. 3 is connected with the onset of full synchrony. Our simulation results also reveal that the minimum in Fig. 3 does not correspond to any change in the system dynamics. 


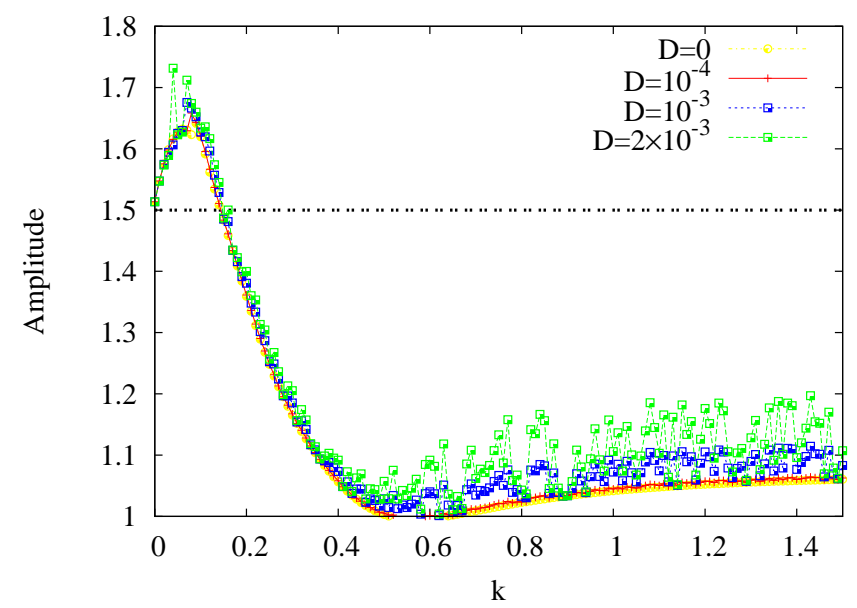

Fig. 3. Amplitude of harmonic oscillation in $x(t)$ as a function of control gain parameter $k$, for the synchronous mode. The dashed horizontal line denotes the uncontrolled case $(k=0)$. and for different noise strength $D$, namely $D=0,10^{-4}, 10^{-3}$ and $2 \times 10^{-3}$, respectively. Other parameters are $\lambda=0.75, \omega=0.5$, and $F_{0}=0.5$.

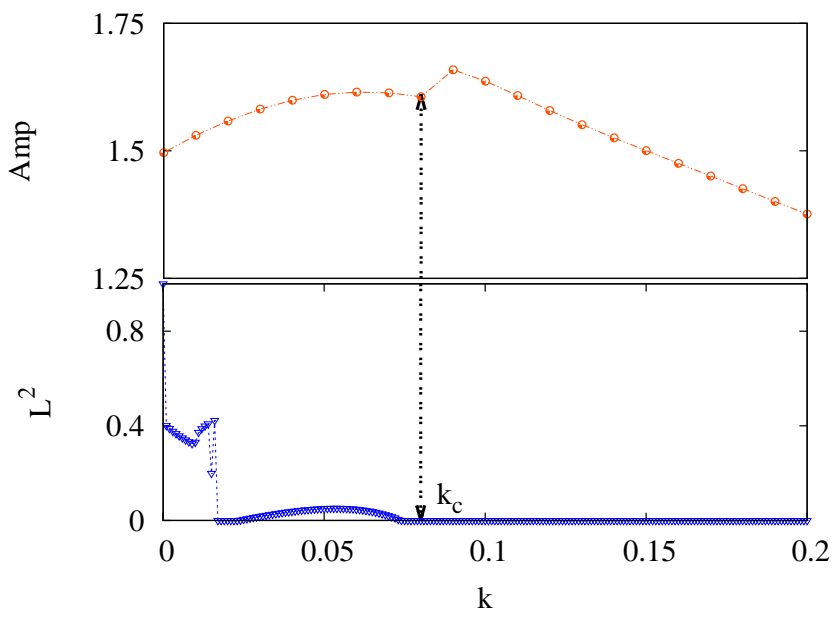

Fig. 4. Synchronization index $L^{2}$ and Amplitude in $x(t)$ as functions of $k$ for the same parameters as in Fig. 3. The critical coupling $(\approx 0.08)$ coincides with the onset of the maximum peak in Fig. 3.

\subsection{Asynchronous mode}

We define the periodic motion satisfying $x(t) \neq y(t)$ as the asynchronous mode. With the synchronous solution, $x(t)=y(t)=s(t)$ move in rhythm as a composite system with state space $s(t)$, each asynchronized system moving independently with the solutions $x(t)$ and $y(t)$, such that the controlled equation in this case satisfies Eq. (3). To consider the effect of the channel on the amplitude of the ratchets, we present in Fig. 5 the amplitude of vibration as a function of the control gain parameter for the same values as in Figs. 3 and 4 . Note that the two ratchets have the same parameters, leading to the same dynamics. Fig. 5 , also shows that there are ranges of $k$ where the amplitude decreases with $k$. For our set of parameters, we find that when $0.1<k<0.24$ and $0.46<k<0.69$, the channel can turn the system in an unfavorable direction where the amplitude of oscillation is decreased.

\subsection{Effects of Noise}

We now consider the effects of noise on our system. We will show that a noisy environment can induce multi-resonance oscillations as well as enhancing the quality of synchronization. In the presence of noise 


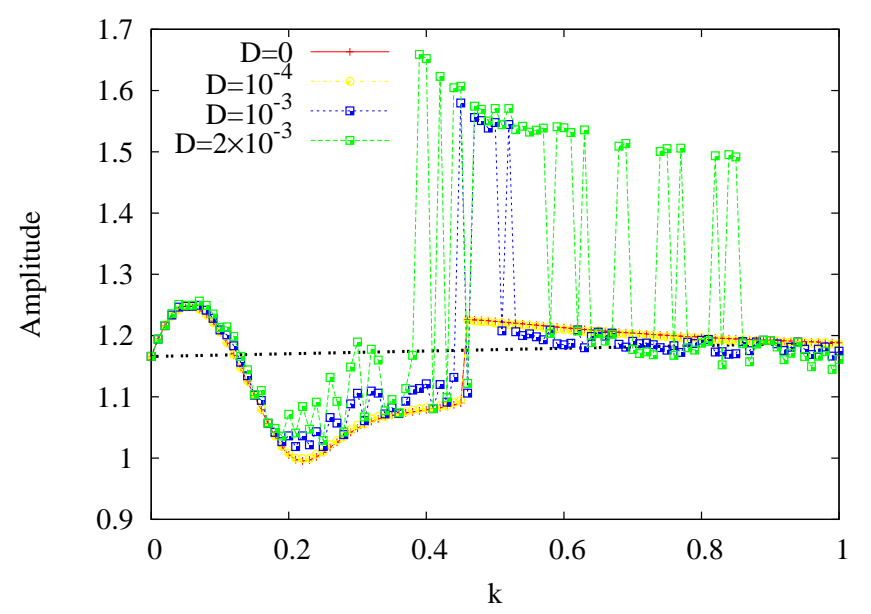

Fig. 5. Amplitude of harmonic oscillation in $x(t)$ as a function of control gain parameter $k$ for the asynchronous mode. The dashed horizontal line denotes the uncontrolled case $(k=0) . \lambda=0.75, \omega=0.5$, and $F_{0}=0.5$.

$(D \neq 0)$, the full synchronization mode is given by the following system

$$
\begin{aligned}
& \ddot{s}+\lambda \dot{s}+\frac{d v(s)}{d s}=F_{0} \cos (\omega t)+k(z-s) \\
& \dot{z}=2 k(s-z)+\xi(t)
\end{aligned}
$$

with $v(s)$ given by equation (2). We now consider separately the induction by noise of multi-resonance and synchronization:

(i) Noise-induced multi-resonance: For $k=0$ and $D=0$ the resonance disappears as shown in Fig. 2. The response of the system is also robust for some very small noise intensities $\left(D \leq 10^{-4}\right)$. However, as the noise intensity approaches $D \approx 0.002$ (see Figs. 3 and 5 ) multiple resonances/multi-resonance oscillations suddenly appear. Figs. 3 and 5 illustrate this phenomenon for different noise intensities and for increasing coupling strengths $k$. Moreover, the multi-resonance appears to be more prominent at larger values of $k$. Further evidence of noise-induced multi-resonance oscillations can be seen in Fig. 6 where the amplitude of harmonic oscillations in $x(t)$ is plotted as a function of $F_{0}$ for two different noise intensities: $D=0$ and $D=0.005$. Remarkably, however, the multi-resonance oscillations are annihilated at larger noise intensities, where the amplitudes exhibit irregular behaviour.

(ii) Noise-enhanced synchronization: Fig. 4 (lower panel) shows that complete synchrony does not occur for weak coupling strength in the absence of noise $(D=0)$. In particular, for $k=0.005$ and $k=0.05, L^{2}$ takes on approximate values of 0.4 and 0.05 , respectively. In this regime, the synchronization can be enhanced by switching on the noise term. To illustrate this, in Fig. 7, we plot the synchronization index $L^{2}$ vs $D$ for $k=0.005$ and $k=0.05$. For $k=0.005$, we see in Fig. 7(a) that for small noise strength, the synchronization performance is not enhanced. However as $D$ rises, the quality of synchronization improves. For $k=0.05$ around the burst prior to the occurrence of stable synchronization for $D=0$, it is evident that noise enhances very significantly the quality of synchronization for almost all $D$ values with some bursts in synchronization being evident (Fig. 7(b)).

In the regime of synchronization, say $k=0.1$ shown in Fig. 3, we also observed that noise assists the complete synchrony between the two ratchets as they interact with the environment (See Fig. 7(c)). These scenarios indicate that noise does not destroy synchronization; rather, synchronization can be enhanced by the presence of the noisy environment. For further increase in $k$ to $k>0.1$ (not shown), the system is globally synchronized for all $k$ and $D$, implying that synchronization also occurs for large $k$ values in the presence of noise. Kenfack \& Singh [2010] recently reported that synchronization would not occur even for large coupling strength where noise enhanced the stochastic dynamics of two coupled underdamped bistable oscillators, due to topological and nondeterministic nature of the systems. Our results here show a 


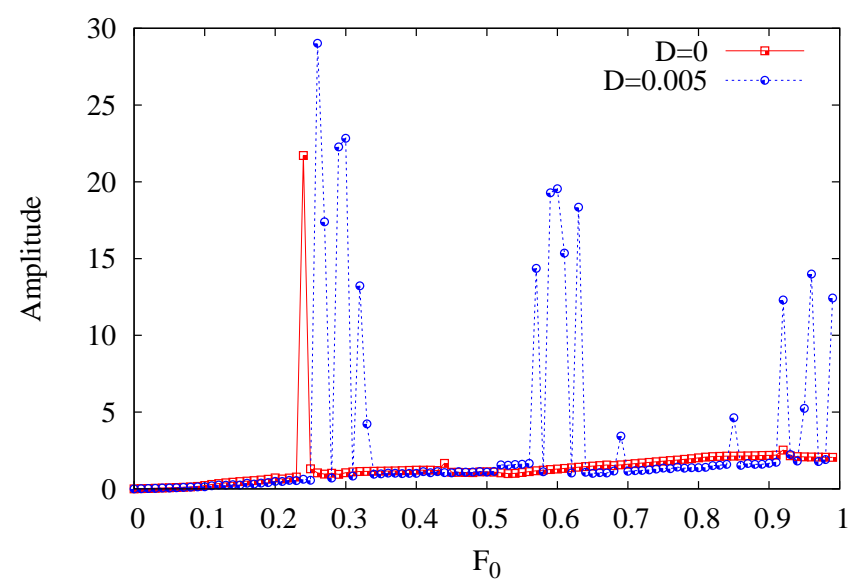

Fig. 6. Amplitude of harmonic oscillation in $x(t)$ as a function of the parameter $F_{0}$ showing the appearance of multi-resonance oscillations for noise intensities $D=0$ and $D=0.005$. The other parameters are: $k=0.5, \lambda=0.75$ and $\omega=0.5$.

contrary situation where noise can indeed enhance and sustain synchronization at large coupling. However, whether this is also applicable to bistable oscillators and in line with stochastic resonance as studied by Kenfack \& Singh [2010] remains an open problem.

To complete this picture, we examine synchronization quality in the parameter space of $D$ and $k$ by estimating the synchronization index $\left(L^{2}\right)$ as functions of both the noise intensity $D$ and the coupling strength $k$. In Fig. $7(\mathrm{~d})$, we have scanned the quantity $L^{2}$ so as to reveal its characteristic behavior and determine the range of noise intensity capable of enhancing synchronization. In general, $L^{2}$ takes on minimum and maximum values of 0 and 1 , respectively. In our calculation, we have truncated the range of values of $L^{2}>0.05$; and, in addition, assumed that when $L^{2} \leq \epsilon=0.005$, the ratchets have reached the complete (strongest) synchronization manifold and that, elsewhere, the systems move away from this manifold. We have identified five regions as denoted by the colorbars, where white and blue are adjacent and the variation runs between the extremes of white and yellow; white is the region where complete synchrony is expected to take place and blue denotes the weakest synchronization regime. From Fig. 7(d), it is evident that the strongest synchronization quality will typically be achieved for $k \leq 0.05$ and for almost all $D$ values. Notice that this corresponds to the weak coupling regime in Fig. 3 where, in the absence of noise the coupled ratchets are not synchronized $\left(L^{2}>0.005\right.$ is the cut-off). Thus, with noisy interaction, the complete synchronization state could be reached in regimes where it was impossible without noise. As we move from the white (strongest synchrony) regime to yellow, we observe the appearance of Arnol'd tongue-like structures at the transition boundaries separating the regions, suggesting that the coupled ratchets could exhibit phase synchronization. The significant of our results is that inertia ratchets, though exhibiting rich transport properties could in addition take advantage of a noisy environment to enhance the conversion of random fluctuations into directed motion in the absence of bias forces.

\section{Control and Optimal Transport}

Consider two identical inertia ratchets coupled via the dynamical environment given by equations (3) and (4). Their dynamics for the synchronous and asynchronous modes in the chaotic regime are exactly the same. To explore the effect of control on the two chaotic ratchets we have simulated them and show in Fig. 8 their phase portrait and the effect of the control gain parameter on their dynamical response. In the region of critical chaos (e.g. $F_{0}=0.0892845, \lambda=0.1$ and $\omega=0.67$ ), the ratchet is highly sensitive to initial conditions, some of which can lead to very different trajectories (see Fig. 8(a) for $k=0$ ). Taking into account the effect of control one can observe that, for $k=0.05$, the system's chaotic regime has given way to a quasiperiodic dynamics (see Fig. 8(b)); and then by further increasing the value of the control gain parameter $k$, it is clear that between $k=0.2$ and $k=0.5$ the system passes through a bi-periodic regime to 

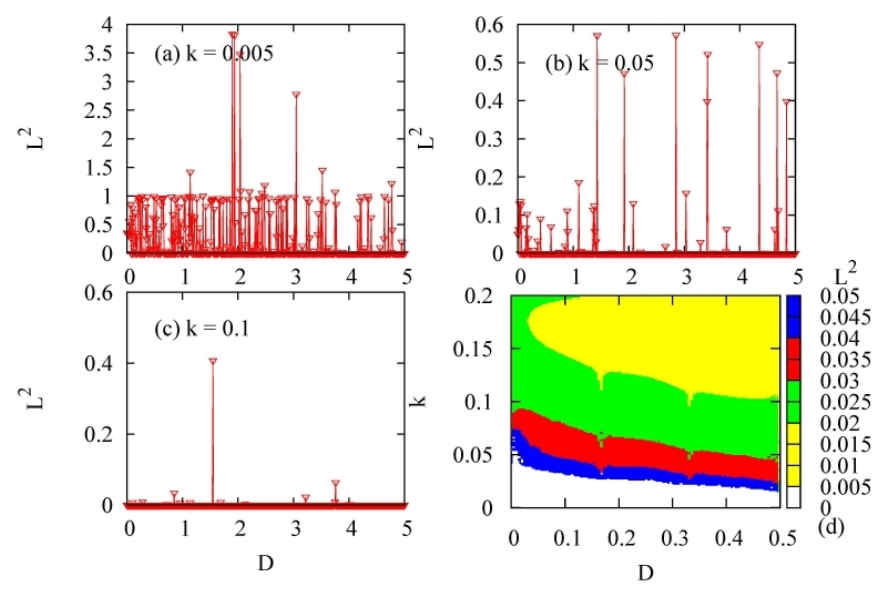

Fig. 7. Synchronization index $L^{2}$ as functions of the noise intensity $D$ showing: (a) weak enhanced synchrony for $k=0.005$, (b) strongly enhanced synchrony for 0.05 and (c) sustained synchrony for 0.1 , respectively. The other parameters are $\lambda=0.75$ and $\omega=0.5$.
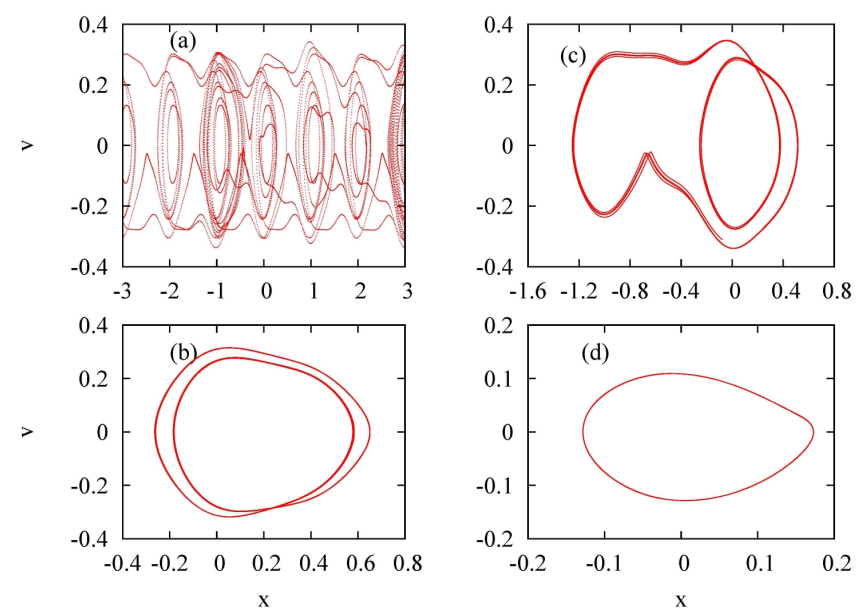

Fig. 8. Phase portraits for increasing control gain parameter. (a) $k=0$ (chaotic orbits), (b) $k=0.05$ (quasiperiodic orbits of period-2), (c) $k=0.2$ (periodic-2 orbit), and (d) $k=0.5$ (periodic- 1 orbit). Note that $D=0$. The case for $D \neq 0$ is same as $D=0$ because the attractors are robust to noisy effects.

a periodic regime and remains periodic thereafter. Thus, the environment suppresses the chaotic dynamics. It can potentially provide an excellent approach for the stabilization of irregular transport fluctuations, including current reversals in the ratchet.

We now turn to the effect of the environment on transport. Here, transport is measured quantitatively by the current $J$, which in turn is defined as the time-average of the average velocity over an ensemble of initial conditions. Over a fixed time [Mateos, 2000; Kenfack et al., 2007] the current in system (5) is defined as

$$
J=\frac{1}{M-n_{c}} \frac{1}{N} \sum_{l=n_{c}}^{M} \sum_{j=1}^{N} \dot{s}^{(j)}\left(t_{l}\right) .
$$

where $N$ is the total number of trajectories, $t_{l}$ the observation time and $M$ the total number of observations. This gives the average velocity, which is then further time-averaged over the number of observations $\left(M-n_{c}\right)$. To ensure that a converged current is obtained, some transient effects are cut-off and this is denoted by $n_{c}$ [Kenfack et al., 2007]. With increasing $k$, the current $J$ is stabilized and reaches a peak value 

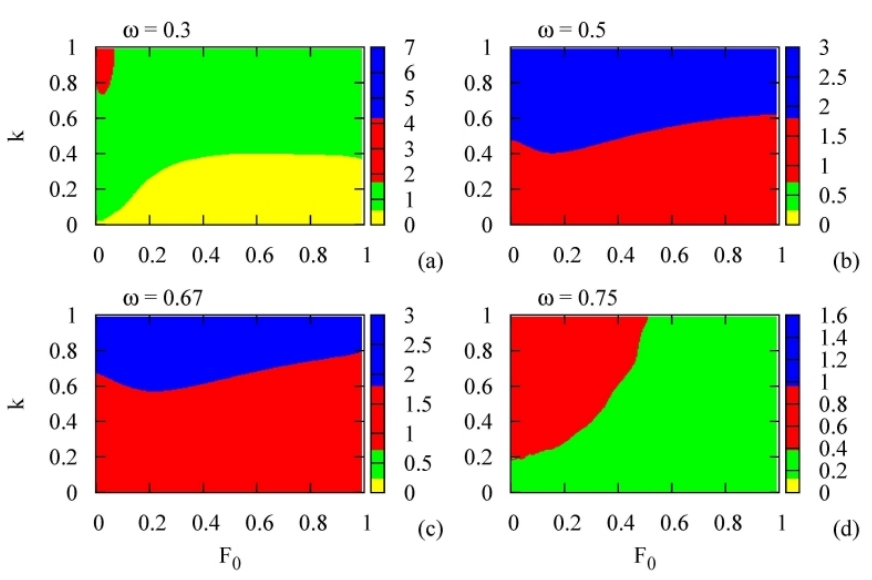

Fig. 9. Current $J_{\max }^{\mu}$ in the units of $10^{-3}$ as function of $k$ and $F_{0}$ showing current enhancement with increasing driving frequency, $\omega . J_{\max }^{\mu}$ has optimum value around $k=1.0$ and within the range $0 \leq F_{0} \leq 0.35$.

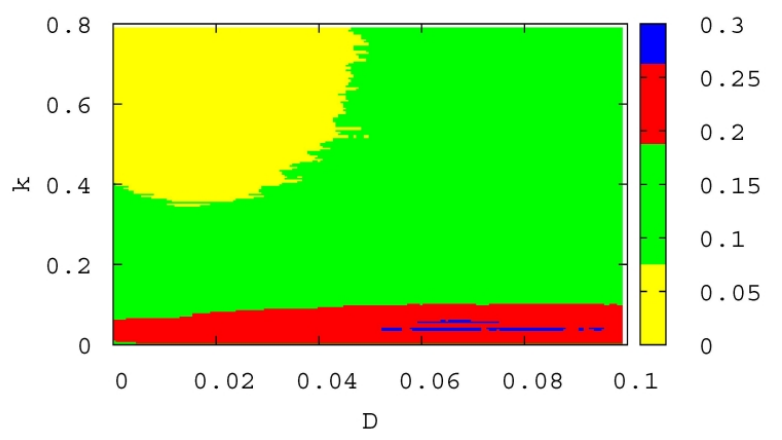

Fig. 10. Current $J_{\text {max }}^{\mu}$ in the units of $10^{-3}$ as function of $D$ and $k$ for $\omega=0.3$ showing regimes of weak, moderate and enhanced transports. $J_{\max }^{\mu}$ has optimum value around weak $k$ and strong $D$; and minimum for larger $k$ and weak $D$.

for fixed $F_{0}$. In view of the irregular fluctuations as well as the multiple current-reversals, it is convenient to define a new quantity, the maximum current computed over a coupling interval $(0 \leq k \leq 1.0)$ and for $0 \leq F_{0} \leq 1.0$. The absolute value of the maximum current is denoted $J_{\max }^{\mu}$.

Figure 9 provides a global view of the effect of the environment on transport in the form of 3D plots for selected driving frequencies. Note that $J_{\max }^{\mu}$ with the four associated colorbars showing different regions, indicating values of $J_{\max }^{\mu}$ as it increases from minimum (yellow) to maximum (blue) are clearly identified. The yellow color denotes regions in which the environment has an insignificant or minimal effect on transport at a given frequency; while the blue color represents the regime where the environment optimizes the magnitude of the current. Starting with $\omega=\omega_{1}=0.3$ (Fig. 9(a)), the optimum value of $J_{\max }^{\mu}$ is $8 \times 10^{-3}$ and occurs within the weak-amplitude or forcing regime, typically $F_{0}<0.1$. If the range of $k$ is further increased to $k=2.0$, we find that the optimum value of $J_{\max }^{\mu}$ is further enhanced, lying within the same $F_{0}$ regime shown in Fig. 9(a). This implies that as the coupled ratchets are driven into a coherent state by their environment, the current is readily amplified.

The parameter regime of current amplification also depends on the frequency $\omega$ of the external driving. For $\omega=\omega_{2}=0.5$, we observe that $J_{\max }^{\mu}$, in the range of $k$ shown takes an optimum value of $6 \times 10^{-3}$ within a broader range of $F_{0}$ which is lower than the optimum value of $J_{\max }^{\mu}$ for $\omega_{1}$ as shown in Fig. 9(b). Further increase in the frequency to moderate values, say $\omega=\omega_{3}=0.67$ as in Fig. 9(c), shows that the forcing amplitude regime for which optimal transport can be achieved is also at larger coupling strength. Moreover, in this moderate $\left(\left(\omega_{2}, \omega_{3}\right)=(0.5,0.67)\right)$ frequency regime, the influence of the environment on transport is more pronounced. However, as the frequency rises, the effect of the environment weakens and 
the behavior seen at low frequencies gradually returns again as shown in Fig. 9(d).

Finally, we demonstrate enhanced transport in the present of noise. Fig. 10 reveals that, in the weakcoupling region where multi-resonances and enhanced synchrony arose due to stochastic effects, the currents also exhibit maxima in the parameter space of $D$ and $k$ for $\omega=0.3$. Again, enhanced current can arise over a broad range of noise intensity. With the combination of large coupling and weak noise intensity, small currents will be obtained; while large coupling strength and large noise intensity will give rise to moderate currents. This same observation was also made for the other frequencies shown in Fig. 9.

\section{Conclusion}

To summarize, we have examined the resonance oscillations and synchronization between two identical ratchets coupled indirectly via a noisy environment. The noisy environment was found to play a significant role in the dynamics and synchronization processes. In the absence of noise and coupling there are of course no resonance oscillations; whereas, when the interaction becomes noisy multi-resonance oscillations are induced. Noise was also found to enhance synchronization quality in the weak-coupling parameter regime where the systems were unsynchronized in the absence of noise, as well as assisting the synchronization performance in the synchronized state where the coupling strength is typically strong. Furthermore, we have demonstrated that the properties of the ratchets can conveniently be altered by changing the feedback gain of the environment, thus, providing an efficient means of controlling the underlying dynamics and the corresponding transport properties. In this way, steady transport can be achieved for appropriately chosen values of the control gain parameter $k$. The control gain regime for optimal transport has also been identified in amplitude-frequency parameter space. For relatively strong coupling, we have shown that optimal transport can be achieved over a broad range of amplitudes for moderate frequencies. The import of our results in general is that useful transport in real macroscopic systems may be enhanced by allowing the system to interact with its environment. Specifically, the rich transport properties of inertia ratchets may significantly be enhanced through the systems' interaction with a noisy environment. Our analysis of the two-ratchet system, leading to synchronized regular motion, could be extended to a system of $n$ identical or nonidentical ratchets whose collective dynamics can be adjusted by playing on the characteristics properties of its environment.

\section{Acknowledgements}

The work of UEV is supported by the British Academy, the Royal Academy of Engineering and the Royal Society of London through the award of a Newton International Fellowship. Part of this work was done at the Max Planck Institute for the Physics of Complex Systems (MPIPKS), Germany during a short research visit there by BRNN. He is grateful to MPIPKS for financial support. The authors are grateful to the anonymous reviewers for their constructive comments.

\section{References}

Al-Khawaja, S. [2005] "Chaotic dynamics of underdamped josephson junctions in a ratchet potential driven by a quasiperiodic external modulation," Physica $C$ 420, 30-36.

Anishchenko, V. S., Astakhov, V., Neiman, A. B., Vadivasova, T. E. \& Schimansky-Geier, L. [2007] Nonlinear Dynamics of Chaotic and Stochastic Systems (Springer, Berlin).

Arizmendi, C. M., Family, F. \& Salas-Brito, A. L. [2001] "Quenched disorder effects on deterministic inertia ratchets," Phys. Rev. E 63, 061104.

Astumian, R. D. [1997] "Thermodynamics and kinetics of a Brownian motor," Science 276, 917-922.

Barbi, M. \& Salerno, M. [2000] "Phase locking effect and current reversals in deterministic underdamped ratchets," Phys. Rev. E 62, 1988-1994.

Camacho, E., Richard, R. \& Howland, H. [2004] "Dynamics of two van der Pol oscillators coupled via a bath," Intern. J. Solids Struct. 41, 2133-2143.

Chen, Z. [2007] "Complete and generalized synchronization in a class of noise-perturbed chaotic systems," Chaos 17, 023106. 
Fischer, I., Vincente, R., Buldu, J. M., Peil, M. \& Mirasso, C. R. [2006] "Zero-lag long-range synchronization via dynamical relaying," Phys. Rev. Lett. 97, 123902.

García-Álvarez, D., Bahraminasab, A., Stefanovska, A. \& McClintock, P. V. E. [2009] "Competition between noise and coupling in the induction of synchronisation," Europhysics Letters 88, 30005.

Hänggi, P. \& Bartussek, R. [1996] "Brownian rectifiers: how to convert Brownian motion into directed transport," Nonlinear Physics of Complex Systems: Current Status and Future, eds. Parisi, J., Muller, S. C. \& Zimmermann, W. (Springer, Berlin), pp. 294-308.

Jülicher, F., Ajdari, A. \& Prost, J. [1997] "Modeling molecular motors," Rev. Mod. Phys. 69, 1269-1281.

Jung, J., Kissner, J. G. \& Hänggi, P. [1996] "Regular and chaotic transport in asymmetric periodic potentials: Inertia ratchets," Phys. Rev. Lett. 76, 3436-3439.

Katriel, G. [2008] "Synchronization of oscillators coupled through an environment," Physica D 237, 29332944.

Kenfack, A. \& Singh, K. P. [2010] "Stochastic resonance in coupled underdamped bistable systems," Phys. Rev. E 82, 0462240.

Kenfack, A., Sweetnam, S. M. \& Pattanayak, A. K. [2007] "Bifurcations and sudden current change in ensembles of classically chaotic ratchets," Phys. Rev. E 75, 056215.

Kostur, M., Hänggi, P., Talkner, P. \& Mateos, J. L. [2005] "Anticipated synchronization in coupled inertia ratchets with time-delay feedback," Phys. Rev. E 72, 036210.

Li, J.-H. [2006] "Chaotic noisy transport of electron pairs in a superconducting junction device: Thermalinertia ratchets," Phys. Rev. E 74, 011114.

Lin, W. \& He, Y. [2005] "Complete synchronization of the noise-perturbed chua's circuits," Chaos 15, 023705.

Lu, P. L., Yang, Y. \& Huang, L. [2008] "Synchronization of linearly coupled networks of deterministic ratchets," Phys. Lett. A 372, 3978-3985.

Maritan, A. \& Banavar, J. R. [1994] "Chaos, noise and synchronization," Phys. Rev. Lett. 72, 1451-1454.

Mateos, J. L. [2000] "Chaotic transport and current reversal in deterministic ratchets," Phys. Rev. Lett. 84, 258-261.

Mateos, J. L. \& Alatriste, F. R. [2008] "Phase synchronization in tilted inertial ratchets as chaotic rotators," Chaos 18, 043125.

Reimann, P., Grifoni, M. \& Hänggi, P. [1997] "Quantum ratchets," Phys. Rev. Lett. 79, 10-13.

Resmi, V., Ambika, G. \& Amritkar, R. E. [2010] "Synchronized states in chaotic systems coupled indirectly through a dynamic environment," Phys. Rev. E 81, 046216.

Rompala, K., Rand, R. \& Howland, H. [2007] "Dynamics of three coupled van der Pol oscillators with application to circadian rhythms," Comm. Nonl. Sc. Numer. Simulat. 12, 794-803.

Schimansky-Geier, L., Abcott, D., Neiman, A. \& van den Broeck, C. (eds.) [2003] Noise in Complex and Stochastic Systems, (Proceedings of SPIE, NM, USA), Vol. 5114 (Bellingham, New Mexico, USA).

Senthilkumar, D. V. \& Kurths, J. [2010] "Characteristics and synchronization of time-delay systems driven by a common noise," Eur. Phys. J. Special Topics 187, 87-93.

Son, W.-S., Kim, I., Park, Y. J. \& Kim, C. M. [2003] "Current reversal with type-I intermittency in deterministic inertia ratchets," Phys. Rev. E 68, 067201.

Steele, C. T., Zivkovic, B. D., Siopes, T. \& Underwood, H. [2003] "Ocular clocks are tightly coupled and act as pacemakers in the circadian system of Japanese quail," Am. J. Physiol. Regul. Integrat. Comp. Physiol. 284, R208-R218.

Strogatz, S. H., Abrams, D. M., McRobie, A., Eckhardt, B. \& Ott, E. [2005] "Crowd synchrony on the millennium bridge," Nature 438, 43-44.

Sun, Y. \& Cao, J. [2007a] "Adaptive synchronization between two different noise-perturbed chaotic systems with fully unknown parameters," Physica A 376, 253-265.

Sun, Y. \& Cao, J. [2007b] "Exponential synchronization of stochastic perturbed chaotic delayed neural networks," Neurocomputing 70, 2477-2485.

Tang, Y., Fang, J. \& Chen, L. [2008] "Lag projective stochastic perturbed synchronization for chaotic systems and its application in secure communication," Int. J. of Modern Phys. B 22, 4175-4188.

Taylor, A. F., Tinsley, M. R., Wang, F., Huang, Z. \& Showalter, K. [2009] "Dynamical quorum sensing 
and synchronization in large populations of chemical oscillators," Science 323, 614-617.

Vincent, U. E., Kenfack, A., Njah, A. N. \& Akinlade, O. [2005] "Bifurcation and chaos in coupled ratchets exhibiting synchronized dynamics," Phys. Rev. E 72, 056213.

Vincent, U. E., Kenfack, A., Senthilkumar, D. V., Mayer, D. \& Kurths, J. [2010a] "Current reversals and synchronization in coupled ratchets," Phys. Rev. E 82, 046208(1-5).

Vincent, U. E. \& Laoye, J. A. [2007a] "Synchronization and anti-synchronization and current transports in non-identical chaotic ratchets," Physica A 384, 230-240.

Vincent, U. E. \& Laoye, J. A. [2007b] "Synchronization and control of directed trasnport in chaotic ratchets via active control," Phys. Lett. A 363, 91-95.

Vincent, U. E., Njah, A. N., Akinlade, O. \& Solarin, A. R. T. [2004] "Phase synchronization in unidirectionally coupled chaotic ratchets," Chaos 14, 1018-1025.

Vincent, U. E., Olusola, O. I., Mayer, D. \& McClintock, P. V. E. [2010b] "Controlling current reversals in synchronized underdamped ratchets," J. Phys. A: Math Theor 43, 165101.

Xu, S. Y., Yang, Y. \& Song, L. [2009] "Control-oriented approaches to anticipating synchronization of chaotic deterministic ratchets," Phys. Lett. A 373, 2226-2236.

Zamora-Munt, J., Masoller, C., Garcia-Ojalvo, J. \& Roy, R. [2010] "Crowd synchrony and quorum sensing in delay-coupled lasers," Phys. Rev. Lett. 105, 264101.

Zarlenga, D. G., Larrondo, H. A., Arizmendi, C. M. \& Family, F. [2009] "Complex synchronization structure of an overdamped ratchet with discontinuous periodic forcing," Phys. Rev. E 80, 011127.

Zhou, C. \& Kurths, J. [2002] "Noise-induced phase synchronization and synchronization transitions in chaotic oscillators," Phys. Rev. Lett. 88, 230602.

Zhou, C., Kurths, J., Kiss, I. Z. \& Hudson, J. L. [2002] "Noise-enhanced phase synchronization of chaotic oscillators," Phys. Rev. Lett. 89, 014101. 